\title{
Coronavirus y anestesia
}

\section{Coronavirus and anesthesia}

\author{
Dr. José Alfonso Ramírez-Guerrero*
}

RESUMEN. La pandemia del nuevo coronavirus COVID-19 ha infectado a más de 800,000 casos en todo el mundo y aún no hay vacuna ni tratamiento específico. Como especialistas en el manejo de la vía aérea, los anestesiólogos estamos expuestos a las secreciones y con un alto riesgo de contraer la infección por COVID-19. En esta revisión se muestran las recomendaciones para disminuir el riesgo a través de técnicas de control de infecciones.

ABSTRACT. The pandemic of the new coronavirus disease, COVID-19 has been involved in more than 800,000 cases worldwide. There isn't vaccine or specific treatment. As specialists in airway management, anesthesiologist are routinely exposed to patients' respiratory secretions and are at high risk of contracting COVID-19 from infected patients. This review provides suggestions on how to minimize this risk by improved infection control.

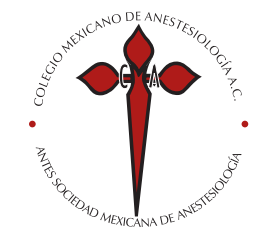

Palabras clave: COVID-19, manejo anestésico, manejo de vía aérea.

Keywords:

COVID-19, anesthesic

management, airway

management.

* Anestesiólogo, Hospital
Médica Sur.

Solicitud de sobretiros:

Dr. José Alfonso

Ramírez-Guerrero

Puente de Piedra Núm. 150, Torre 2,

Consultorio 611, Col.

Toriello Guerra,

14050, Alcaldía de Tlalpan,

Ciudad de México.

E-mail: alfonsodoc@yahoo.com.mx

Recibido para publicación:

31-03-2020

Aceptado para publicación: 08-04-2020
L a pandemia del nuevo coronavirus (COVID-19) ha alcanzado a todos los continentes y el número de víctimas continúa en ascenso. La cifra de nuevos casos y muertes cambia de manera acelerada en todo el mundo. Al momento de escribir estas líneas hay 838,000 casos con 41,261 muertos. En México las cifras oficiales reportan 1,094 casos con 29 fallecidos. Sin embargo, el número de casos podría ser muy superior si consideramos el número de pruebas realizadas por cada 100 mil habitantes (0.3 en México, 410 en Corea del Sur). Hemos entrado en la fase 2, en la que los casos de contagios se dan dentro de la propia comunidad (dispersión comunitaria). Para cuando ustedes lean estas líneas podríamos estar en la fase 3 o de epidemia, como sucede ya en otros países (Italia, EUA, España).

El propósito de este trabajo es presentar las medidas de seguridad prudentes en el perioperatorio. La rapidez con que se ha extendido esta pandemia ha impedido realizar estudios metodológicamente rigurosos, por lo que muchas recomendaciones están basadas en experiencias de otras pandemias (Síndrome Respiratorio Agudo Grave [SARS], Síndrome Respiratorio de
Oriente Medio [MERS]) o de recomendaciones de países que se han visto afectados antes que nosotros por el COVID-19(1).

Características patogénicas: el COVID-19 pertenece al grupo de los betacoronavirus, familia del coronavirus (incluyen al SARS-Cov, MERS-Cov). Tiene un diámetro de 60-140 nm con espigas de 9-12 nm. Es sensible a la luz y puede ser inactivado a $56{ }^{\circ} \mathrm{C}$, con etil-éter, etanol al $75 \%$, peróxido de hidrógeno, desinfectantes de cloro y cloroformo. La clorhexidina es menos eficaz ${ }^{(2)}$.

Epidemiología: el virus se transmite por la inhalación de microgotas en aerosol que expulsan los pacientes sintomáticos o asintomáticos, en particular si se está cerca (menos de 1.5 metros) y llegan a tener contacto con las mucosas. La viabilidad del virus en el aerosol es de tres horas. También puede ocurrir por contacto directo con superficies contaminadas en las que el COVID-19 tiene una supervivencia variable: cobre cuatro horas, cartón 24 horas, acero 48 horas y plástico 72 horas, con la subsecuente autoinoculación ${ }^{(3)}$.

Esto hace que los trabajadores de la salud (TS), incluidos los anestesiólogos, intensivistas y enfermeras, tengan un ries- 
go elevado por estar en contacto con el aerosol respiratorio, secreciones y otros líquidos corporales durante los procedimientos para el manejo de la vía aérea. En China se reportaron al 11 de febrero de 2020 1,716 TS infectados con COVID-19 de un total de 44,762 casos $^{(4)}$. Durante la epidemia del SARS, en Ontario, Canadá, 51\% de los casos eran TS y aquéllos que habían participado en procedimientos de intubación tenían un riesgo 13 veces mayor de adquirir el SARS ${ }^{(5,6)}$.

Precauciones perioperatorias: si el personal labora en dos instituciones, uno con alta concentración de casos y otro que no los tenga, debería realizar su trabajo en uno de los dos para evitar infecciones cruzadas. Todos deben recibir entrenamiento en el uso de equipo de protección personal (EPP): cómo limpiarlo, desinfectarlo, guardarlo e inspeccionarlo para detectar daños. Las embarazadas y el personal inmunosuprimido no debe estar en contacto con pacientes COVID-19.

1. Evaluación preoperatoria ${ }^{(1,6)}$ : las siguientes recomendaciones son para casos ya diagnosticados o con alta sospecha de estar infectados. Dado que existen reportes de transmisión de pacientes infectados asintomáticos, se recomienda el uso de cubrebocas en la evaluación preoperatoria de todos los pacientes mientras la epidemia esté en curso (Tabla 1).

- El personal médico debe portar EPP que incluye: bata desechable, guantes, protección ocular, gorros y cubrebocas quirúrgico o mascarillas N95.

- Los pacientes deben entrar al consultorio uno por uno para minimizar el contacto con otros pacientes o con el personal médico.

- Se les debe medir la temperatura con termómetros electrónicos, si es mayor a $37.3^{\circ} \mathrm{C}$ deberá ser acompañado a la clínica de control de infecciones.

- El anestesiólogo debe tomar una historia clínica detallada y exploración detallada de los campos pulmonares.

- Después del contacto con el paciente el médico debe lavarse las manos con agua y jabón o limpiarlas con peróxido de hidrógeno al 2-3\%.
- Ante la sospecha de infección con COVID-19 (aún con temperatura normal) el paciente debe ser acompañado a la clínica de control de infecciones.

- Al final del turno, el consultorio deberá ser limpiado y desinfectado en todas las superficies, muebles y equipo con peróxido de hidrógeno al 2-3\%.

2. Preparación para cirugía de emergencia ${ }^{(1,7)}$ :

- Todo paciente que requiera cirugía de urgencia debe completar la evaluación previa.

- Si no es una cirugía urgente deberá posponerse.

- Si es una urgencia el paciente deberá ser puesto en un área de aislamiento y transferirse al quirófano dedicado para casos con COVID-19.

- Si se sospecha o se confirma que es un caso con COVID-19 deberá ser reportado a la clínica de control de infecciones del hospital y deberá ser transferido al hospital designado para estos casos si su condición lo permite.

- El paciente deberá ser trasladado directamente al quirófano por una ruta designada y el personal médico que lo acompañe debe portar respiradores N95, protección ocular (goggles o tiendas faciales), gorros, guantes y bata.

3. Manejo anestésico:

- El equipo de anestesia debe comunicar a la clínica de infecciones que un paciente con COVID-19 será transferido al quirófano designado para esos casos.

- El quirófano debe contar con sistemas de presión negativa. De no haber, los sistemas de presión positiva y/o el aire acondicionado deben permanecer apagados.

- El quirófano exclusivo para estos casos debe tener letreros en la puerta que así lo indique. Sólo podrá entrar el personal encargado del caso. Se debe evitar abrir las puertas del quirófano para no contaminar el corredor.

- Se usará una máquina de anestesia destinada a ese quirófano. Más adelante se explica cómo desinfectar.

- El filtro del circuito (o nariz artificial) debe instalarse entre el tubo endotraqueal y la parte distal del circuito,

Tabla 1: Precauciones de contacto.

Instalación del paciente

Control de tráfico

Equipo

Diseño de ingeniería

Higiene de manos

Guantes

Bata

Mascarillas

Protección respiratoria

Protección ocular

Cobertura de la cabeza
Habitaciones dedicadas para casos sospechosos o confirmados COVID-19 con aislamiento

Limitar el personal presente durante los procedimientos al esencial. Las puertas deben permanecer cerradas y las entradas y salidas minimizadas

Se usará de preferencia equipo desechable. El resto deberá limpiarse y desinfectarse

Sistemas de succión cerrado para la succión de vía aérea

Lavarse con agua y jabón y después usar sanitizadores basados en alcohol

Después de higiene manual colocarse guantes limpios no estériles

Batas largas, limpias, no estériles y resistentes al agua

Mascarillas quirúrgicas

Mascarillas N95 o equivalentes

Goggles o caretas faciales desechables que cubran el frente y lados de la cara

Diferentes a los gorros quirúrgicos. Deben proteger toda la cabeza, cuello y hombros 
Tabla 2: Protección en escala ascendente para los Trabajadores de la Salud.**

\begin{tabular}{|c|c|c|c|c|}
\hline Escala & Rutina & Nivel I & Nivel II & Nivel III \\
\hline & Pisos regulares & $\begin{array}{l}\text { Pisos para } \\
\text { enfermedades } \\
\text { infecciosas }\end{array}$ & $\begin{array}{l}\text { Cuidados sin } \\
\text { contacto pacientes } \\
\text { diagnosticados o } \\
\text { sospecha COVID-19 }\end{array}$ & $\begin{array}{l}\text { Contacto directo } \\
\text { con pacientes } \\
\text { diagnosticados o } \\
\text { sospecha COVID-19 }\end{array}$ \\
\hline Cubrebocas & + & + & - & - \\
\hline Máscara N95 & - & - & + & + \\
\hline Protección ocular & - & - & + & + \\
\hline Higiene de manos & + & + & + & + \\
\hline Guantes & + & + & + & + \\
\hline Bata de protección & - & + & $+-^{*}$ & - \\
\hline Traje de protección & - & - & $+-^{*}$ & + \\
\hline Gorro quirúrgico & - & + & + & + \\
\hline Protección de cabeza & - & - & - & + \\
\hline Cubrecalzado & - & - & + & + \\
\hline
\end{tabular}

** Basado en las regulaciones establecidas en el Hospital Tongji en Wuhan.

+ Obligado, - No necesario, +- Decisión de acuerdo al escenario de trabajo, +-* Elección entre bata o traje de protección de acuerdo a los recursos locales para el nivel III.

y otro en la interfase del circuito y la máquina. Deberá cambiarse cada 3-4 horas en cirugías prolongadas.

- La secuencia sugerida para la instalación del EPP es: lavarse las manos, colocar el uniforme y gorro; lavarse las manos, colocar cubrebocas, guantes, protección ocular, protección de calzado, bata y segundo juego de guantes; probar que todo el equipo esté bien ajustado en los otros integrantes del equipo y acceder al quirófano.

4. Tipos de anestesia: experiencias previas con el SARS en China demostraron que la intubación endotraqueal fue un factor de riesgo independiente para la diseminación nosocomial y el contagio a los TS.

Aunque hay recomendaciones para los procedimientos que generan aerosol (PGA), si el caso lo permite, habrá que seleccionar la anestesia regional ${ }^{(8)}$.

- Anestesia regional o neuroaxial: el paciente debe ser evaluado, bloqueado y recuperado dentro del quirófano designado para los casos COVID-19. El paciente deberá portar una máscara N95 o al menos un cubrebocas quirúrgico todo el tiempo. Sólo el equipo y fármacos necesarios se tendrán dentro de la sala para evitar desperdicios, ya que TODO deberá desecharse al final del procedimiento. Equipo y fármacos adicionales que pudieran necesitarse deberán estar fuera del quirófano al alcance con una enfermera o médico disponible en todo momento (evitar tiempos perdidos en ir hasta la central de equipos). Si se usa un ultrasonido deberá ser cubierto con bolsas de plástico. Si el paciente no tiene trastornos pulmonares para la oxigenación podrá emplearse sedación, de lo contrario es preferible no usarse para evitar que se deteriore la oxigenación. En caso de bloqueo neuroaxial, se debe estar pendiente si el bloqueo motor asciende a niveles que interfieran con los músculos accesorios de la ventilación que pondría en riesgo a pacientes con dificultad respiratoria previa. Para evitar la aerosolización y el riesgo de contaminación, no se recomienda el uso de ventilación con presión positiva no invasiva, cánulas de alto flujo de $\mathrm{O}_{2}$ ni mascarillas de $\mathrm{O}_{2}$ con sistemas Venturi. La dispersión del aire exhalado se incrementa a mayor flujo de $\mathrm{O}_{2}: 0.2,0.22,0.3$ y $0.4 \mathrm{~m}$ con flujos de 4,6 , 8 y $10 \mathrm{~L} / \mathrm{min}$, y esta distancia aumenta con la tos ${ }^{(9)}$. Sólo se podrán emplear puntas de $\mathrm{O}_{2}$ por debajo de la máscara N95 o del cubrebocas al menor flujo que permita mantener la $\mathrm{SpO}_{2}>90 \%$.

- Anestesia general: intubar a un paciente con COVID-19 implica riesgos para los TS. En el Hospital Zhongnan en Wuhan 40 de 138 casos hospitalizados eran TS con transmisión intrahospitalaria como mecanismo probable de infección y ocurrieron antes de establecerse las medidas de protección. A partir del seguimiento de esas medidas no observaron más casos ${ }^{(10)}$.En la epidemia del SARS, aunque el apego a las guías de protección ayudó a disminuir el riesgo, no lograron evitarlo por completo. En la Tabla 1 se muestran las precauciones de contacto y en la Tabla 2 los diferentes niveles de protección de acuerdo al tipo de pacientes que se manejen.

- Todo anestesiólogo que vaya a estar en contacto con un paciente COVID-19 debe portar EPP de nivel III. Todos deben recibir entrenamiento en el uso de equipo de protección personal: cómo limpiarlo, des- 
infectarlo, guardarlo e inspeccionarlo para detectar daños ${ }^{(10)}$.

- Antes de la inducción, corroborar que todo el personal tenga bien colocado el EPP. ES PRIORITARIA SU PROTECCIÓN Y LA DEL RESTO DEL EQUIPO. Se recomienda la intubación con secuencia rápida: preoxigenar adecuadamente, usar una dosis de relajante que asegure una parálisis completa para evitar que tosa durante la intubación que deberá realizarla el miembro con más experiencia para evitar más de un intento. Se recomienda el videolaringoscopio para mayor éxito y para alejar nuestro rostro de la vía aérea del paciente. Con el uso del broncoscopio podemos incrementar la distancia operador-vía aérea y disminuir el riesgo. El EPP necesario para estos casos puede dificultar el procedimiento, por lo que es recomendable entrenar con maniquís antes de usarlos por primera vez. Deberá evitarse la intubación despierto, ya que la tos o el anestésico local atomizado genera aerosolización de partículas. En caso de necesitar ventilación manual, emplear volúmenes corrientes bajos, sellar bien la mascarilla facial y colocar un filtro de alta eficiencia (capaces de remover $99.97 \%$ de las partículas mayores a 0.3 micras) entre la mascarilla y el circuito. Se prefiere el uso de tubo endotraqueal (TET) a la máscara laríngea, ya que el TET sella de forma más hermética la vía aérea. Asegure una exhalación completa con la máscara facial bien sellada a la cara del paciente antes de quitarla para proceder a la laringoscopía. Después de intubar, inflar bien el globo del TET y sólo hasta después iniciar la ventilación con presión positiva. De manera inmediata poner la hoja del laringoscopio dentro de doble bolsa de plástico. Posteriormente hay que evitar las desconexiones del circuito, si esto no es posible, permitir la exhalación completa, pinzar el TET y desconectar el menor tiempo posible. Este mismo procedimiento se recomienda cuando el paciente tiene que pasar intubado a la Unidad de Terapia Intensiva (UTI). El equipo usado deberá limpiarse y desinfectarse con óxido de etileno o peróxido de hidrógeno ${ }^{(1,7,10)}$.

- Recuperación: antes de la extubación se recomienda cubrir la nariz y boca con gasas húmedas para disminuir la exposición a las secreciones. Deberá extubarse dentro del quirófano tratando de evitar que tosa. No será trasladado a la sala de recuperación, sino directamente a un cuarto aislado con presión negativa, a través de un pasillo y elevador exclusivo para estos casos y con un cubrebocas o una mascarilla N95. El personal que acompañe al paciente deberá cambiar toda la ropa. Inmediatamente después, los pasillos y el elevador deben limpiarse y desinfectarse. Si el paciente pasa intubado a la UTI deberá usarse un Ambu desechable ${ }^{(1,7,10)}$.
5. Cuidado del equipo postanestesia y manejo de desechables:

- Todo el equipo deberá ser desechable y usado para cada caso. Se colocará en doble bolsa de plástico, etiquetado como «caso COVID-19», se sellarán herméticamente y se rociaran con desinfectante clorinado.

- El personal médico y de enfermería deberá retirarse todo el equipo antes de salir del quirófano que se colocará en dobles bolsas de plástico selladas. El orden sugerido es: retirar cobertura de zapatos y guantes; lavar manos; quitar goggles/máscara facial; lavar manos; retirar gorro; lavar manos; baño completo y colocar ropa personal.

- El equipo de anestesia debe ser limpiado y desinfectado con peróxido de hidrógeno al 2-3\% o desinfectante de cloro al 2-5\%. El absorbedor de $\mathrm{CO}_{2}$ debe cambiarse por completo después de cada caso. La máquina de anestesia y el ventilador deben desarmarse y desinfectarse a alta temperatura o con peróxido de hidrógeno al 2-3\%.

- El quirófano lavado y desinfectado con peróxido de hidrógeno al 2-3\%, alcohol al 75\% o solución de cloro de 2-5 g/L. El personal que limpie el quirófano debe portar el mismo equipo que el personal médico ${ }^{(1,11)}$.

6. Intubación traqueal fuera de quirófano: el número de pacientes que desarrollan insuficiencia respiratoria es alto. Muchos son intubados en las salas de emergencia o en las UTI, en donde el riesgo de exposición para el personal es mayor, ya que las condiciones de intubación pueden ser subóptimas, pero deberán seguirse los mismos lineamientos que para la anestesia general ${ }^{(10,12,13)}$.

- Anticipe la intubación cuando un paciente se deteriore de su función respiratoria. Lleva varios minutos aplicar todas las barreras de precaución.

- Preoxigenar durante cinco minutos para realizar de preferencia una intubación con técnica de secuencia rápida. Por ser pacientes que ya están en insuficiencia respiratoria, el tiempo de apnea que toleran antes de caer en hipoxemia es más corto. Si se necesitan flujos altos de $\mathrm{O}_{2}$ antes de intubar, tratar de sellar la máscara facial al paciente, para evitar el incremento en la fuga del aerosol.

- La intubación deberá realizarla el anestesiólogo de más experiencia para minimizar el número de intentos. Se recomienda el uso de videolaringoscopio o fibroscopio para aumentar la distancia entre la cavidad oral y el médico que hace el procedimiento.

- Se tratará de evitar la intubación despierto. Aunque apliquemos lidocaína en spray o en inhalaciones corremos el riesgo de que el paciente tosa durante la intubación. Si predecimos una vía aérea difícil debemos contar con todo el equipo necesario para una intubación exitosa.

- Se debe instalar un filtro de alta eficiencia entre la máscara y el circuito o el Ambu. 
- La aspiración oral o traqueal debe hacerse con un sistema de succión cerrado después de la intubación.

7. Vigilancia del personal médico que ha estado en contacto con pacientes COVID-19: cualquier trabajador de la salud que haya estado en contacto con un caso, sospechoso o confirmado de COVID-19, que desarrolle fiebre, tos o fatiga, deberá informarlo a las autoridades sanitarias del hospital para realizar estudios más completos (proteína C reactiva, TAC de tórax) y mantenerse en aislamiento.

\section{REFERENCIAS}

1. Chen X, Liu Y, Gong Y, Guo X, Zuo M, Li J, et al. Perioperative Management of Patients Infected with the Novel Coronavirus: Recommendation from the Joint Task Force of the Chinese Society of Anesthesiology and the Chinese Association of Anesthesiologists. Anesthesiology. 2020 Mar 26. doi: 10.1097/ ALN.0000000000003301.

2. Peng PWH, Ho PL, Hota SS. Outbreak of a new coronavirus: what anaesthetists should know. Br J Anaesth. 2020 Feb 27. pii: S00070912(20)30098-2. doi: 10.1016/j.bja.2020.02.008.

3. Wong J, Goh QY, Tan Z, Lie SA, Tay YC, Ng SY, et al. Preparing for a COVID-19 pandemic: a review of operating room outbreak response measures in a large tertiary hospital in Singapore. Can J Anaesth. 2020 Mar 11. doi: 10.1007/s12630-020-01620-9.

4. Meng L, Qiu H, Wan L, Ai Y, Xue Z, Guo Q, et al. Intubation and ventilation amid the COVID-19 outbreak: Wuhan's experience. Anesthesiology. 2020 Mar 26. doi: 10.1097/ ALN.0000000000003296.

5. Bowdle A, Munoz-Price LS. Preventing infection of patients and healthcare workers should be the new normal in the era of Novel Coronavirus Epidemics. Anesthesiology. 2020 Mar 25. doi: 10.1097/ ALN.0000000000003295.

6. Ti LK, Ang LS, Foong TW, Ng BSW. What we do when a COVID-19 patient needs an operation: operating room preparation and guidance. Can J Anaesth. 2020 Mar 6. doi: 10.1007/s12630-020-01617-4.
7. Wax RS, Christian MD. Practical recommendations for critical care and anesthesiology teams caring for novel coronavirus (2019-nCoV) patients. Can J Anaesth. 2020 Feb 12. doi: 10.1007/s12630-020-01591-x.

8. Lie SA, Wong SW, Wong LT, Wong TGL, Chong SY. Practical considerations for performing regional anesthesia: lessons learned from the COVID-19 pandemic. Can J Anaesth. 2020 Mar 24. doi: 10.1007/ s12630-020-01637-0.

9. Peng PW, Wong DT, Bevan D, Gardam M. Infection control and anesthesia: lessons learned from the Toronto SARS outbreak. Can J Anaesth. 2003; 50 (10): 989-997.

10. Tran K, Cimon K, Severn M, Pessoa-Silva CL, Conly J. Aerosol generating procedures and risk of transmission of acute respiratory infections to healthcare workers: a systematic review. PLoS One. 2012; 7 (4): e35797. doi: 10.1371/journal.pone.0035797.

11. van Doremalen N, Bushmaker T, Morris DH, Holbrook MG, Gamble A, Williamson BN, et al. Aerosol and Surface Stability of SARS-CoV-2 as Compared with SARS-CoV-1. N Engl J Med. 2020 Mar 17. doi: 10.1056/ NEJMc2004973.

12. Peng PW, Wong DT, Bevan D, Gardam M. Infection control and anesthesia: lessons learned from the Toronto SARS outbreak. Can J Anaesth. 2003;50: 989-997.

13. Wax RS, Christian MD. Practical recommendations for critical care and anesthesiology teams caring for novel coronavirus (2019-nCoV) patients. Can J Anaesth. 2020. doi: 10.1007/s12630-020-01591-X. 